\title{
Practical Applications of Swarm Intelligence and Evolutionary Computation
}

\section{Sukumar Senthilkumar}

School of Computing Science and Engineering, Vellore Institute of Technology University, India

*Corresponding author: Sukumar Senthilkumar, School of Computing Science and Engineering, Vellore Institute of Technology University, Vellore-632014, Tamilnadu, India, Tel: 91-416 2202811; E-mail:senthilkumar@vit.ac.in

Received date: March 13, 2014, Accepted date: March 13, 2014, Publication date: March 22, 2014

Copyright: ( 2014 Senthilkumar S. This is an open-access article distributed under the terms of the Creative Commons Attribution License, which permits unrestricted use, distribution, and reproduction in any medium, provided the original author and source are credited.

\section{Editorial}

It is essential to have new computing techniques and algorithms because the computer evolution has changed human societies for communication, transportation, industrial production, administration, writing, and bookkeeping, technological advances/science, entertainment etc. It is well known that some problems cannot be solved through conventional hardware and software due to some drawbacks. Computing tasks have to be well defined, fairly predictable, and computable in reasonable time with serial computers in case of traditional techniques. In order to overcome such cumbersome, there are some alternatives such as DNA based computing (chemical computation), Quantum computing (quantum physical computation), Bio-computing (simulation of biological mechanisms) are proposed by various researchers. Swarm intelligence has gained very high priority among the researchers from different field like commerce, science and engineering etc. Recently much attraction is devoted for advanced, innovative and interdisciplinary research developments involving the theoretical, experimental and practical aspects of the two paradigms which are carried out in the fields of nature-inspired intelligent computation based on the principle of swarm intelligence and evolutionary algorithm. In order to tackle and solve existing real world complex problems, researchers have formulated by looking into natural processes and creatures both as model and metaphor. Due to the fact that, there has been remarkable growth in the field of natureinspired search and optimization algorithms which are widely and extensively applied to a variety of problems, ranging from scientific research to industry and commerce. In specific, the two major families of algorithms that primarily constitute this area today are the evolutionary computing methods and the swarm intelligence algorithms. Even though both families of algorithms are generally dedicated towards solving search and optimization problems, they are certainly not equivalent, and each has its own distinguishing features. It is true that, reinforcing each other's performance makes powerful hybrid algorithms capable of solving many intractable search and optimization problems. The concept of swarm intelligence is based on the collective social behavior of decentralized body, either natural or artificial like ant colonies, fish, bird flocks, honey bee etc. In other words swarm intelligence computation technique is one of the recent and advanced research topics in the field of artificial intelligence which is concerned with the design of intelligent multi agent systems by taking inspiration from the collective behaviors of social insects and other animal societies. They are characterized by a decentralized way of working that mimics the behavior of the swarm. Swarm Intelligence is a successful paradigm for the algorithm with many complex real life problems.

Any swarm intelligence and other related evolutionary algorithms (EAs) provide a framework for effectively sampling large search spaces, and the basic technique is both broadly applicable and easily tailored to specific problems. The overall notions of evolutionary algorithms are computer programs that attempt to solve many complex problems by mimicking the processes of Darwinian evolution. It is to be noted that in an EA a number of artificial creatures search over the space of the problem. But, they compete continually with each other to discover optimal areas of the search space. On the other hand it is hoped that over time the most successful of these creatures will evolve to discover the optimal solution. It is certain that evolutionary computations have a number of components, procedures, or operators that must be specified in order to successfully execute a specific evolutionary algorithm. The predominant components are representation (definition of individuals), evaluation function (or fitness function), population, parent selection mechanism, variation operators, recombination and mutation, survivor selection mechanism (replacement). Each of the components is specified in order to define a particular evolutionary algorithm. Moreover, in order to yield a running algorithm the initialization procedure and a termination condition must also be defined. For instance, the eight queen's problem i.e. in a regular black and white chess board $(8 \times 8)$ eight queens that must place on the board in such a way that no two queens can check each other. It is possible in general that, it can be extended to n-queens problem also with the same notion. Many classical artificial intelligence approaches to this problem work in a constructive, or incremental, fashion. Initially, one starts with placing one queen and after having placed $n$ queens, one attempts to place the $(n+1)$ queen does not check any others. Sometimes, the end user need to have a backtracking mechanism in such a way that there is no feasible position for the ( $\mathrm{n}$ +1 ) th queen, the nth is moved to another position. The other example is the knapsack problem or 0-1 knapsack problem, a generalization of many industrial problems. Given a set of $\mathrm{n}$ of items, each of which has some value vi attached to it and cost ci for selecting a subset of those items that maximizes the value while keeping the summed cost with some capacity Cmax. Therefore, when packing a back-pack for a "round the world" trip, one must balance likely utility of the items they wish to take against the fact that they have limited volume (the items chosen must fit in one bag) and weight (airlines impose fees for luggage over a given weight).

In the recent past decades, biologists and natural scientists have been investigating the behaviors of social insects because of the amazing efficiency of these natural swarm systems. A swarm is a large number of homogenous, simple agents interacting locally among themselves, and their environment, with no central control to allow a global interesting behavior to emerge. Swarm based algorithms have recently emerged as a family of nature inspired, population based algorithms that are capable of producing low cost, fast, and robust solutions to several complex problems. Swarm intelligence is an algorithm or a device, which is designed for solving distributed 
Citation: Sukumar Senthil kumar (2014) Practical Applications of Swarm Intelligence and Evolutionary Computation. Int J Swarm Intell and Evol Comput 3: e106. doi: 10.4172/2090-4908.1000e106

Page 2 of 2

problems. It was illumined by the social behavior of gregarious insects and other animals. There are many novel application areas where nature-inspired intelligent computation is involved such as Communication Engineering, Internet Computing, Mathematical Modeling, Computational Biology, Aerospace, Systems and Control, Sensors, Nonlinear statistical and Applied Physics, Power Systems, Environmental Modeling and Software, Robotics and Control, Future Computing Devices, Mathematical and Computational Psychology, Bioinformatics, Structural and Mechanical Designs, Cognitive Neuroscience, Financial Services and Engineering, Business Intelligence, Operations Research and Decision Sciences, Pattern Recognition, Image Processing and Machine Vision, Brain-computer Interfacing, Management Information Systems, Civil, Chemical, Aeronautical, Computational Chemistry, Manufacturing, Biomedicine and Biochemistry, etc.

It is pertinent to pin point out that nature has rich inspirational source and there is still much more to learn from them and it is endless. It is possible that one can take advantage of the social collective behavior of swarms to solve our real world problems, by observing how these swarms have survived and solved their own challenges in nature. Several simple agents interacting locally among themselves can eventually emerge a sophisticated global behavior. Different swarm intelligence based computational models are rapidly growing, as they are generally computationally inexpensive, robust, and simple. Swarm intelligence based optimization techniques are far reaching in many domains, and have a wide range of successful applications on different diversified areas. Though swarm intelligence is an active field in artificial intelligence and emerging; computing, and its potential is still far from being exhausted, with many studies are exponentially growing and going on still. Finally, it is left to the choice of the end user to decide which swarm intelligence and evolutionary computation have to be employed to solve real world complex problem successfully which may become a very effective aspect of artificial and computational intelligence in near future. 